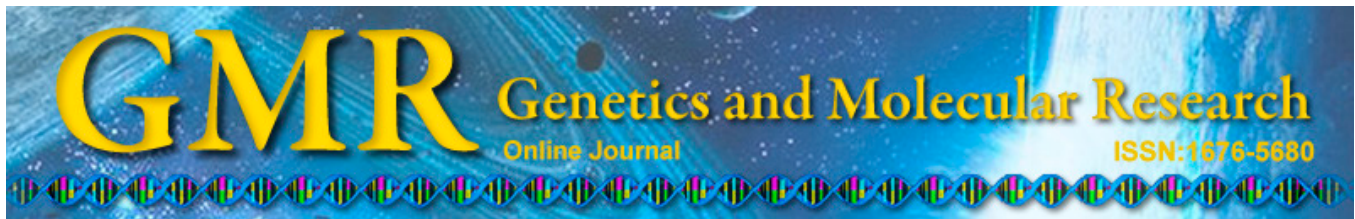

\title{
Effect of hypothalamic-pituitary-adrenal axis alterations on glucose and lipid metabolism in diabetic rats
}

\author{
M.W. Si ${ }^{1}$, M.K. Yang ${ }^{2}$ and X.D. Fu ${ }^{1}$ \\ ${ }^{1}$ Liaocheng People's Hospital, Liaocheng, Shandong Province, China \\ ${ }^{2}$ Ranping People's Hospital, Ranping, Shandong Province, China \\ Corresponding author: M.W. Si \\ E-mail: simingwenlph@126.com
}

Genet. Mol. Res. 14 (3): 9562-9570 (2015)

Received December 4, 2014

Accepted April 7, 2015

Published August 14, 2015

DOI http://dx.doi.org/10.4238/2015.August.14.19

\begin{abstract}
This study investigated the relationship between alterations in the hypothalamic-pituitary-adrenal (HPA) axis function and glucose and lipid metabolism in diabetic rats. To accomplish this a diabetes model was established by jointly administering a longterm high-fat diet plus Streptozotocin (STZ; $50 \mathrm{mg} / \mathrm{kg}$ ip). The rats were randomly divided into four groups: 1) a normal control group, 2) a model group, 3) astragalus polysaccharide (APS) group, and 4) a metformin group. APS and metformin hydrochloride were administered intragastrically $\left(100 \mathrm{mg} \cdot \mathrm{kg}^{-1} \mathrm{~d}^{-1}\right)$. Rat blood glucose and body weight were measured once per week, and urine was collected for $24 \mathrm{~h}$ after 30 days of administration of APS. The levels of blood lipids, insulin, and corticosterone (CORT), as well as hypothalamic CRH, pituitary $\mathrm{ACTH}$, urine sugar and CORT were measured. Compared with the normal control group, the levels of blood sugar, urine sugar, TC, and TG significantly increased in the model group, and the levels of hepatic glycogen and HDL-C decreased. Administration of APS was shown to reverse these changes. Furthermore, as compared with the normal control group, the levels of insulin and hypothalamic CRH in the model
\end{abstract}


group decreased significantly, while the levels of plasma ACTH and CORT, pituitary ACTH, and urine CORT were elevated. Again, APS administration improves these outcomes and returns their levels to normal. Thus, the glucose and lipid metabolic disorder in the highfat diet and STZ-induced diabetes model may be related to increased HPA axis activity. The hypoglycemic effect of the traditional Chinese medicine, ASP, may improve HPA axis functioning and aid in the treatment of diabetes.

Key words: Streptozotocin; HPA axis; Astragalus polysaccharide; Diabetes; Glucose and lipid metabolism

\section{INTRODUCTION}

Diabetes mellitus (DM) is a disease that is a serious threat to human health, but its etiology and pathogenesis has not been fully elucidated. With respect to DM pathogenesis, it was proposed the doctrine of neuroendocrine immune-modulation (NIM), where the balance and stability of the internal environment is maintained by the interaction, intercoordination, and mutual restraint of the nervous system, endocrine system, and immune system. A defect in glucose metabolism is the most obvious feature of diabetes. Glucose metabolism is directly or indirectly linked to the hypothalamic-pituitary-adrenal (HPA) axis and other important axes in the NIM network (Rouch et al., 2012; Marzi et al., 2013). Streptozotocin (STZ), an antibiotic extracted from Streptomyces, is composed of glucose molecules and a methylated nitrogen source, which is one of the drugs commonly used in producing diabetic animal models. Specifically, several low-dose injections of STZ, which is highly toxic to pancreas islet $\beta$-cells, can eventually lead to fulminant type 1 diabetes. The pathogenesis induced is similar to the pathogenesis of clinical type 1 diabetes, indicating that STZ can be transported by the lowaffinity glucose transporter proteins (GLUT2) of the pancreas islet $\beta$-cells and cause structural damage and insulin secretory dysfunction (Weiss et al., 2012). In this paper, a diabetic rat model was jointly induced by a high-fat diet plus low-dose STZ to study the functional changes in the HPA axis and the regulatory effect of ASP.

\section{MATERIAL AND METHODS}

\section{Experimental animals}

Female, sterile Wistar rats weighed $200 \pm 20 \mathrm{~g}$ were provided by the Experimental Animal Center of Beijing University [SCXK (Beijing) 2011-0012].

\section{Drugs and reagents}

STZ was purchased from Hengyuan Biotechnology Co., Ltd, Shanghai, China. Astragalus polysaccharide was bought from Zhongguan Jianye Biotechnology Co., Ltd., Henan, China. Metformin chloride was obtained from Jingfeng Pharmaceutics Co., Ltd., Beijing, China (batch No.: 20120516). Blood sugar, TC, TG, HDL-C detection kits and ACTH, CRH 
and CORT ELISA kits were purchased from Wako Pure Chemical Industries, Ltd, Shandong, China. An insulin radioimmunoassay kit was purchased from Jinbiao Biotechnology Co., Ltd., Shanghai, China.

\section{Apparatus}

Semi-automatic Biochemical Analyzer and Radioimmunoassay C-counter was purchased from Rayto (USA). Electronic balance, ultraviolet-visible spectrophotometer, and high-speed refrigerated centrifuge were bought from Adventurer (USA).

\section{Modeling, grouping, and administration}

Female Wistar rats were fed a high-fat diet for $30 \mathrm{~d}$, which consisted of $5 \%$ egg yolk, $15 \%$ sucrose, $15 \%$ lard, and $65 \%$ basal diet. An ip injection of STZ (50 mg/kg) was then administered after the rats had fasted for $12 \mathrm{~h}$ to induce diabetes. Blood glucose was measured 5 days after injection of STZ, and the rats whose blood glucose values were greater than $15 \mathrm{mM}$ were selected for the experiment. The rats were randomly divided into four groups: 1) a normal control group (non-diabetic mice), 2) a diabetes model group, 3) an APS administration group (100 $\left.\mathrm{mg} \cdot \mathrm{kg}^{-1} \mathrm{day}^{-1}\right)$, and 4) a metformin administration group $\left(100 \mathrm{mg} \cdot \mathrm{kg}^{-1} \mathrm{day}^{-1}\right)$. The normal control and model groups were given equal volumes of distilled water. The above injections were administrated intragastrically (ig). Blood sugar and body weight were measured weekly. Thirty days after continuous administration, urine was collected using metabolic cages. After the last administration, the rats were fasted and fed water for $12 \mathrm{~h}$ and then decapitated to collect trunk blood, which was centrifuged at 3000 $\mathrm{rev} / \mathrm{min}$ at $4^{\circ} \mathrm{C}$ for $15 \mathrm{~min}$. The plasma was reserved separately in different centrifuge tubes and stored at $-25^{\circ} \mathrm{C}$ for later use.

\section{Determination of blood sugar, urine sugar, blood lipid, and liver glycogen levels}

Blood glucose and lipids were determined in accordance with the manufacturer instructions, and hepatic glycogen was detected by anthrone colorimetry, the detailed method has been reported previously (Sun et al., 2013).

\section{Determination of insulin, CRH, ACTH, and CORT levels}

Insulin was measured by radioimmunoassay, and $\mathrm{CRH}, \mathrm{ACTH}$, and CORT were measured by sandwich ELISA. The total amount of CORT in the 24-h urine was calculated as follows:

$$
\text { Urine CORT }=\text { urine CORT concentration } \mathrm{x} \text { urine volume }
$$

\section{Statistical analysis}

All data were analyzed by SPSS 12.5 and reported as means \pm SD. Significant differences were determined by the Student $t$-test 


\section{RESULTS}

\section{Blood sugar, urine sugar, liver glycogen and blood lipid levels}

The blood sugar of the rats that received APS or metformin was significantly lower than the model group 30 days after administration $(\mathrm{P}<0.05)$, with the APS group showing a $20.9 \%$ decrease as compared with the model group (Figure 1). Thirty days after administration, the urine glucose concentration of the model group was 6.86 times that of the normal control group $(\mathrm{P}<0.01)$, and the concentration of urine glucose in the APS and metformin groups were significantly lower as compared with the model group $(\mathrm{P}<0.01)$ (Figure 2). Moreover, the hepatic glycogen level of the rats in the model group was significantly less than that of the normal control group $(\mathrm{P}<0.05)$. Although APS and metformin could improve the level of hepatic glycogen, no statistically difference was found (Figure 3 ). With respect to blood lipids, the level of TC was elevated, and HDL-C was depressed in the model group as compared to the normal control group $(\mathrm{P}<0.01$ and $\mathrm{P}<0.05$, respectively). The level of HDL-C in the APS group was significantly higher than that of the model group $(\mathrm{P}<0.05)$ (Figure 4$)$.

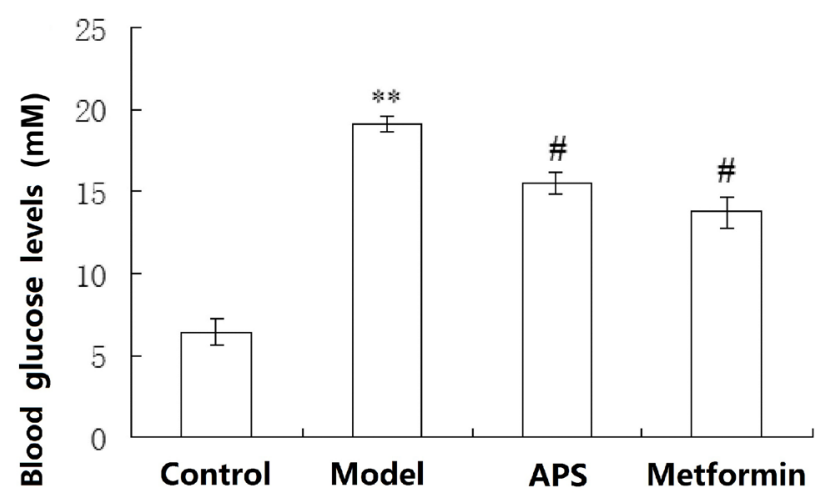

Figure 1. Blood sugar levels 30 days after drug intervention $(\mathrm{mM}) .{ }^{* *} \mathrm{P}<0.01$ vs control; ${ }^{*} \mathrm{P}<0.05$ vs model.

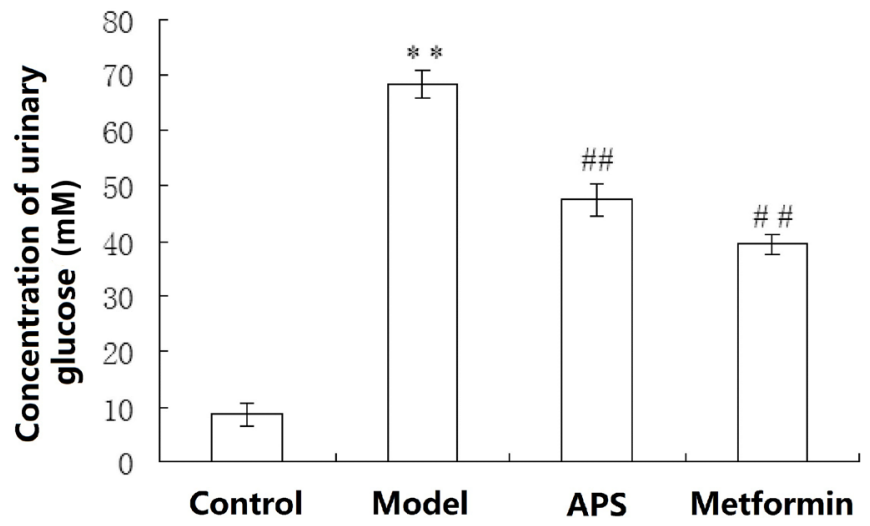

Figure 2. Urine sugar levels 30 days after drug intervention $(\mathrm{mM}) .{ }^{* *} \mathrm{P}<0.01$ vs control; ${ }^{\#} \mathrm{P}<0.01$ vs model. 


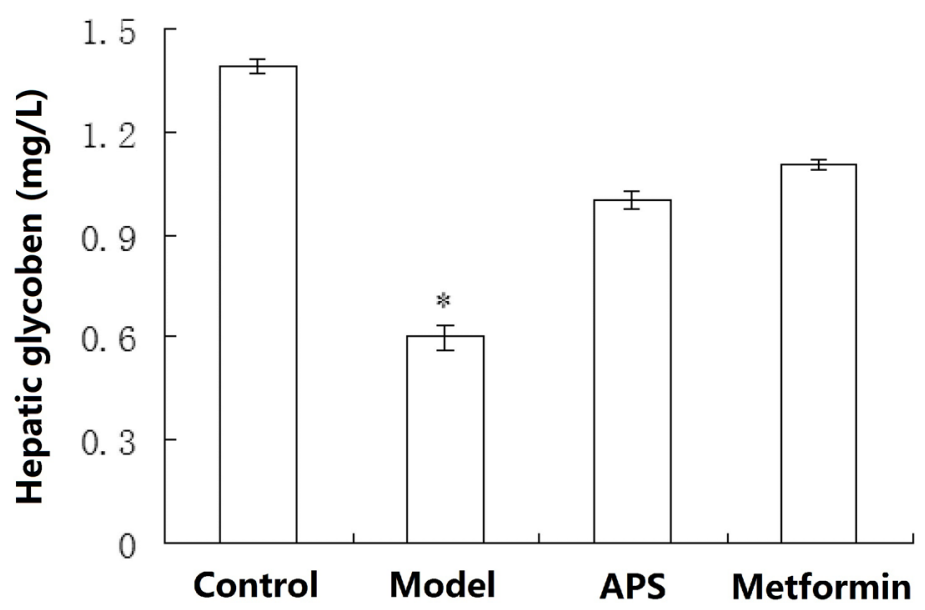

Figure 3. Liver glycogen levels 30 days after drug intervention $(\mathrm{mg} / \mathrm{L}) .{ }^{*} \mathrm{P}<0.05$ vs control.

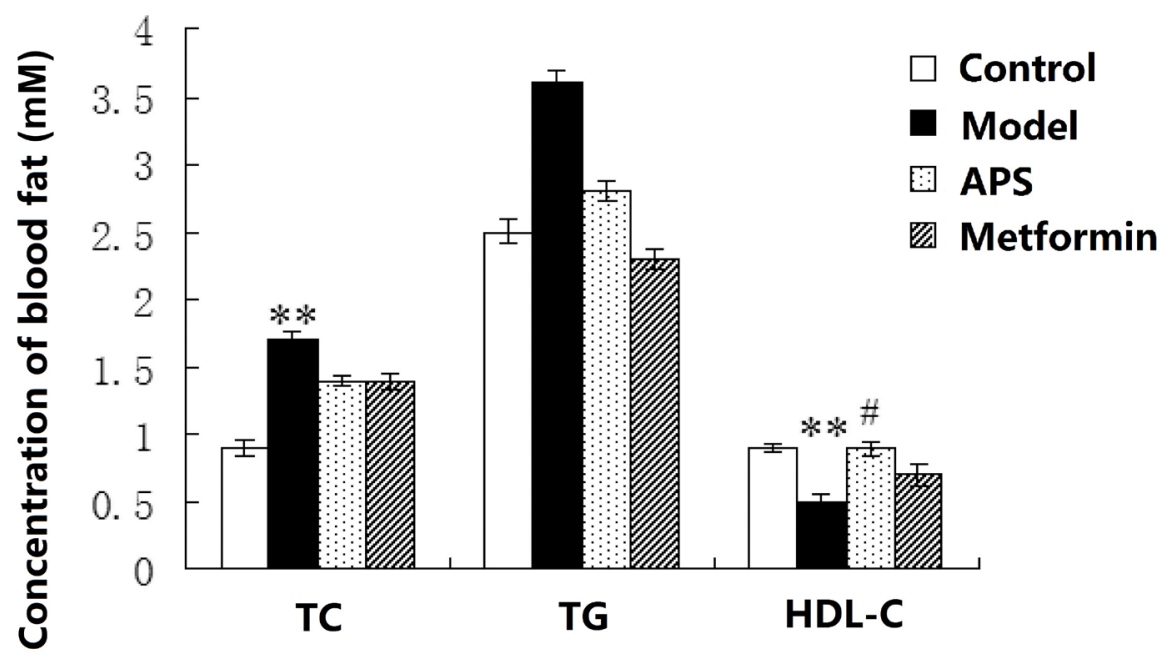

Figure 4. Blood lipid levels (mM). ${ }^{* *} \mathrm{P}<0.01$ vs control; ${ }^{*} \mathrm{P}<0.05$ vs model.

\section{Insulin and HPA axis hormone levels}

The insulin level was significantly lower in the model group, and significantly higher in the APS and metformin groups as compared to the normal control group $(\mathrm{P}<0.05)$. There was no significant change in the plasma CRH level of all groups. The plasma ACTH and CORT levels of the model group were higher than the normal control group $(\mathrm{P}<0.05)$. APS and metformin administration reversed this trend, and lowered ACTH and CORT levels significantly, as compared with the model group $(\mathrm{P}<0.05)$ (Figure 5). Additionally, the hypotha- 
lamic $\mathrm{CRH}$ level in the model rats was $56.49 \%$ lower than that of the normal rats $(\mathrm{P}<0.05)$. APS and metformin treatment tended to increase $\mathrm{CRH}$, but the differences were not statistically significant. The pituitary ACTH level was the highest in the model group [i.e., 48.13\% higher than that of the normal group $(\mathrm{P}<0.05)]$. This level decreased significantly in both APS and metformin groups $(\mathrm{P}<0.05)$ (Figure 6). The CORT level in 24-h urine was the highest in the model group $(\mathrm{P}<0.01)$, and APS and metformin reversed this effect $(\mathrm{P}<0.05)$ (Figure 7).

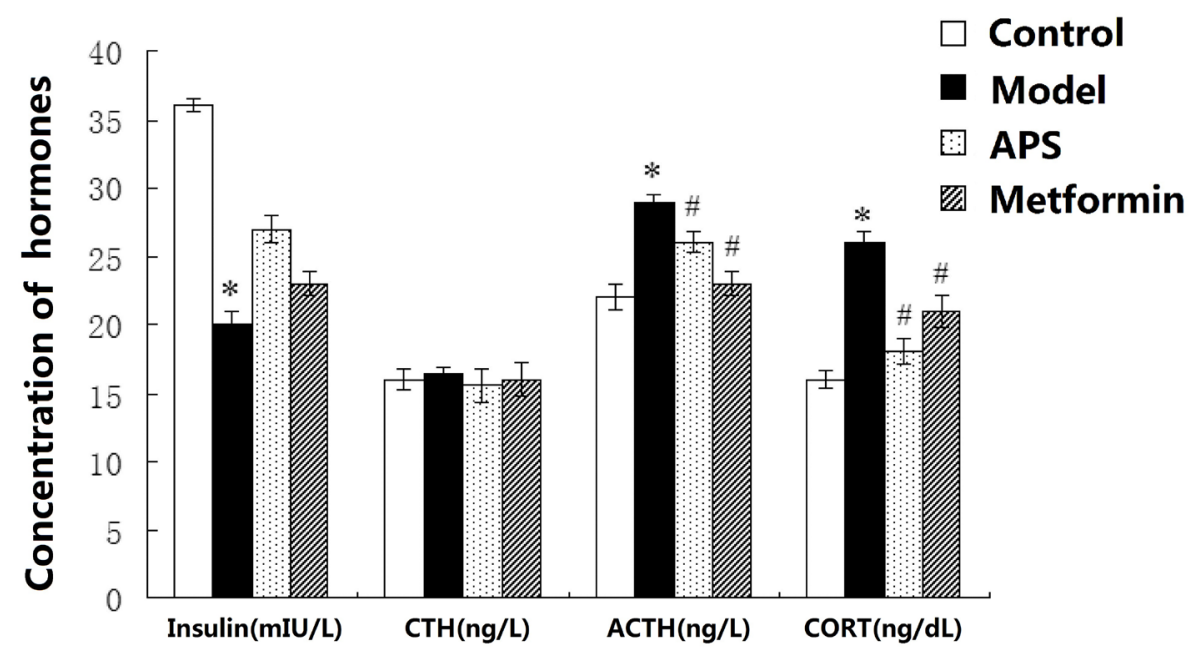

Figure 5. Insulin, CTH, ACTH, and CORT levels in blood. ${ }^{*} \mathrm{P}<0.05$ vs control; ${ }^{*} \mathrm{P}<0.05$ vs model.

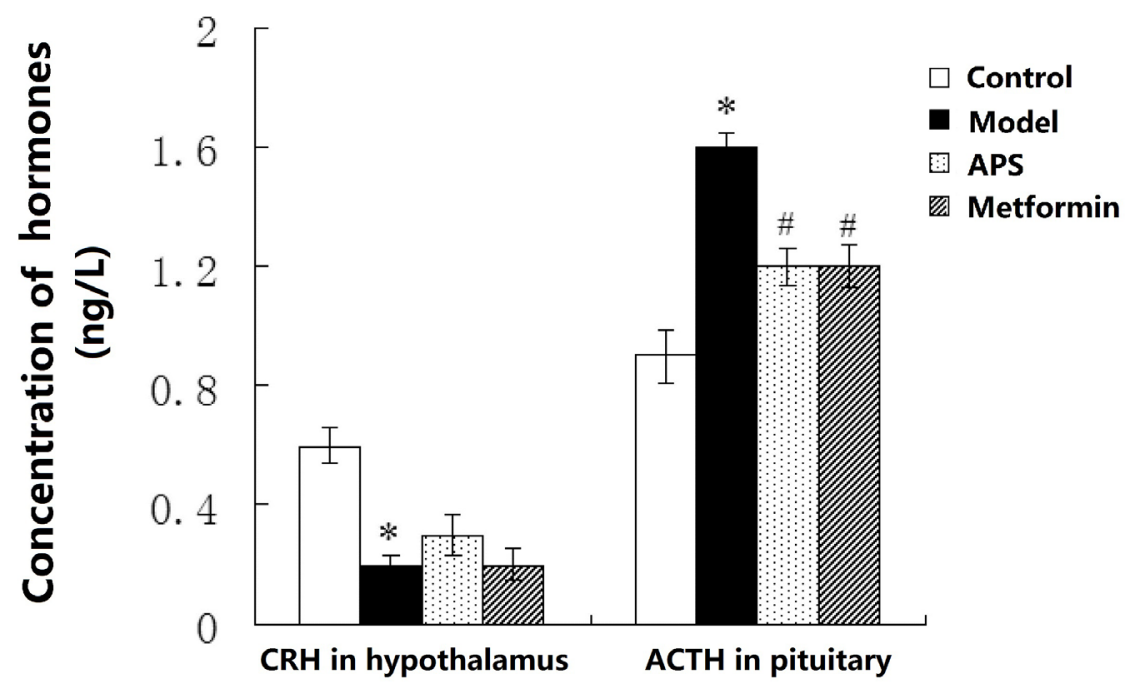

Figure 6. Hypothalamic CRH levels and hypothalamus and pituitary ACTH levels (ng/L). ${ }^{*} \mathrm{P}<0.05$ vs control; ${ }^{\#} \mathrm{P}<0.05$ vs model. 


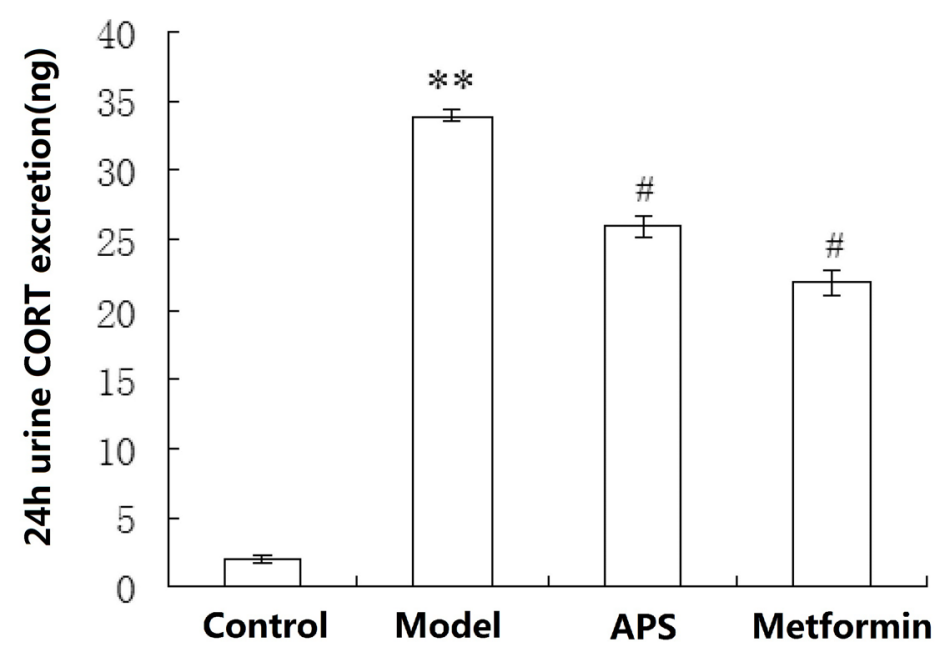

Figure 7. CORT levels in urine within 24 hafter the 30 days of treatment (ng). ${ }^{*} * \mathrm{P}<0.01$ vs control; ${ }^{*} \mathrm{P}<0.05$ vs model.

\section{DISCUSSION}

Diabetes is a common disease, but the mechanism of its pathogenesis has not been fully elucidated yet. The neuroendocrine-immune regulatory network not only plays an important role in maintaining internal homeostasis, but is also involved in the regulation of aging, stress, and many other pathophysiological processes (Kumar et al., 2012; Osuji et al., 2012; Wilbur, 2012). There is evidence that the incidence of diabetes is related to neuroendocrine-immune network dysfunction, and in particular, dysfunction in the HPA axis (Cui and Li, 2012; Sumin et al., 2012; Arredondo, 2013). The HPA axis is an important feedback regulation system that participates in the regulation of glucose metabolism. Thus, changes in HPA axis functioning might play a key role in the incidence and development of diabetes (Cuthbertson et al., 2012; Mercader et al., 2012).

This study has indicated that defects in glucose and lipid metabolism occurred as a result of changes in the HPA axis functioning in diabetic rats. The pituitary and plasma levels of ACTH in the rats of the diabetes model group were significantly higher than that of the normal control group (Figures 5 and 6). Increased ACTH could further promote adrenal cortex secretion of CORT, so as to elevate CORT in the blood. Indeed, excessive CORT was excreted through the urine, and the 24-h CORT excretion of rats in the diabetic model group was significantly larger than that of the normal group (Figure 7). Although the urine CORT concentration in the model group was not significantly different from that of the normal control group, the 24-h urine excretion of diabetic rats was ten times or a hundred times of that of normal rats. $\mathrm{CRH}$ is the main substance that promotes the secretion of pituitary ACTH, but the experimental results indicated that plasma $\mathrm{CRH}$ in the diabetes model group did not change significantly as compared to the normal control group (Figure 5). This may be due to the high concentration of plasma ACTH and CORT, which inhibits CRH secretion and makes the levels observed here appear normal. The results also showed that $\mathrm{CRH}$ in the hypothalamus decreased as compared with the normal rats (Figure 6), which is mainly because the CRH secreted by the 
paraventricular nucleus in the hypothalamus is not the only source of plasma CRH. Studies have shown that peripheral tissues such as the pancreas, adrenal gland, and small intestine are also important sources of plasma CRH (Nishioka et al., 1994). The hypothalamus-secreted $\mathrm{CRH}$ accounts for only a small portion of the plasma CRH.

Given that STZ causes damage to pancreatic $\beta$-cells and reduces the secretion of insulin, we inferred that in this model, blood sugar might increase initially, followed by the elevated activity of the HPA axis. Metformin reduces blood glucose in diabetic rats by promoting the utilization of glucose by peripheral tissues (Figure 1), with the corresponding decline of the HPA axis hormones (i.e., ACTH, CRH) (Figures 5 and 6). Therefore, HPA axis activity is likely to be reduced with decreasing of blood glucose. Chan and Lotspeich (1962) used phlorizin (a compound that increases urine glucose excretion) in the treatment of diabetic rats, which allowed for the fasting blood glucose to return to normal, but postprandial glucose remained mildly elevated. Moreover, the phlorizin treatment could not make the HPA axis activity of diabetic rats return to normal, which strongly suggests that chronic hyperglycemia itself is not the reason for increased HPA axis activity in diabetic rats. Elevated HPA axis activity will increase the production of CORT so as to increase CORT in the plasma. Excessive CORT may promote lipolysis, gluconeogenesis, and decreased glucose uptake by cells, thus weakening the role of insulin in regulating blood sugar, and causing or aggravating tissue insulin resistance (Campbell-Thompson et al., 2012; Lee et al., 2012). This shows that the elevated blood sugar and increased HPA axis activity in the diabetes model are mutually reinforcing, which increased blood sugar and HPA axis activity accelerate the deleterious effects of diabetes. APS can effectively reduce blood glucose to normal levels (Figures 1,2, and 3), and at the same time can decrease ACTH and CORT in the plasma and tissues (Figures 5 and 6), so that glucose metabolism is further improved.

Based on the above results, we suggest that the defects in glucose and lipid metabolism in our diabetes model, which was jointly induced by a high-fat diet plus low-dose STZ, is related to changes in the HPA axis. The traditional Chinese medicine APS can significantly improve glucose and lipid metabolism in diabetic rats, and the mechanism by which this occurs may be associated with decreased activity in the HPA axis.

\section{Conflicts of interest}

The authors declare no conflict of interest.

\section{REFERENCES}

Arredondo A (2013). Diabetes: a global challenge with high economic burden for public health systems and society. Am. J. Public Health 103: e1-e2.

Campbell-Thompson M, Wasserfall C, Montgomery EL, Atkinson MA, et al. (2012). Pancreas organ weight in individuals with disease-associated autoantibodies at risk for type 1 diabetes. JAMA 308: 2337-2339.

Chan SS and Lotspeich WD (1962). Comparative effects of phlorizin and phloretin on glucose transport in the cat kidney. Am. J. Physiol. 203: 975-979.

Cui TH and Li YY (2012). Treatment of type 2 diabetes mellitus oral Chinese patent medicine literature metrology analysis. Zhongguo Zhong Yao Za Zhi 37: 2649-2652.

Cuthbertson DJ, Irwin A, Gardner CJ, Daousi C, et al. (2012). Improved glycaemia correlates with liver fat reduction in obese, type 2 diabetes, patients given glucagon-like peptide-1 (GLP-1) receptor agonists. PLoS One 7: e50117. Doi: 10.1371/journal.pone.0050117. 
Kumar KH, Pandey I, Singh K and Mukherjee D (2012). Unusual presentation of renal tuberculosis in type 2 diabetes. Niger. J. Clin. Pract. 15: 498-499.

Lee J, Lee SH, Hur KY, Woo SY, et al. (2012). Statins and the risk of gastric cancer in diabetes patients. BMC Cancer 12: 596.

Marzi C, Huth C, Herder C, Baumert J, et al. (2013). Acute-phase serum amyloid A protein and its implication in the development of type 2 diabetes in the KORA S4/F4 study. Diabetes Care 36: 1321-1326.

Mercader JM, Puiggros M, Segrè AV, Planet E, et al. (2012). Identification of novel type 2 diabetes candidate genes involved in the crosstalk between the mitochondrial and the insulin signaling systems. PLoS Genet. 8: e1003046. Doi: 10.1371/journal.pgen.1003046.

Nishioka T, Iyota K, Takao T, Suemaru S, et al. (1994) Plasma CRH response to water immersion-restraint stress in rats bearing a hypothalamic knife cut. Endocr. J. 41: 453-459.

Osuji CU, Nzerem BA, Dioka CE and Onwubuya EI (2012). Metabolic syndrome in newly diagnosed type 2 diabetes mellitus using NCEP-ATP III, the Nnewi experience. Niger. J. Clin. Pract. 15: 475-80.

Rouch I, Roche F, Dauphinot V, Laurent B, et al. (2012). Diabetes, impaired fasting glucose, and cognitive decline in a population of elderly community residents. Aging Clin. Exp. Res. 24: 377-83.

Sumin AN, Bezdenezhnykh NA, Bezdenezhnykh AV, Ivanov SV, et al. (2012). Effect of type 2 diabetes mellitus on extent of multifocal atherosclerosis in patients with ischemic heart disease. Kardiologiia 52: 33-41.

Sun G, Lin X, Shen L, Wu F, et al. (2013) Mono-PEGylated radix ophiopogonis polysaccharide for the treatment of myocardial ischemia. Eur. J. Pharm. Sci. 49: 629-636.

Weiss CO, Boyd CM, Wolff JL and Leff B (2012). Prevalence of diabetes treatment effect modifiers: the external validity of trials to older adults. Aging Clin. Exp. Res. 24: 370-376.

Wilbur K (2012). Diabetes Treatment Satisfaction Questionnaire - An Arabic adaptation for Qatar. Diabetes Res. Clin. Pract. 99: e24-e26. 